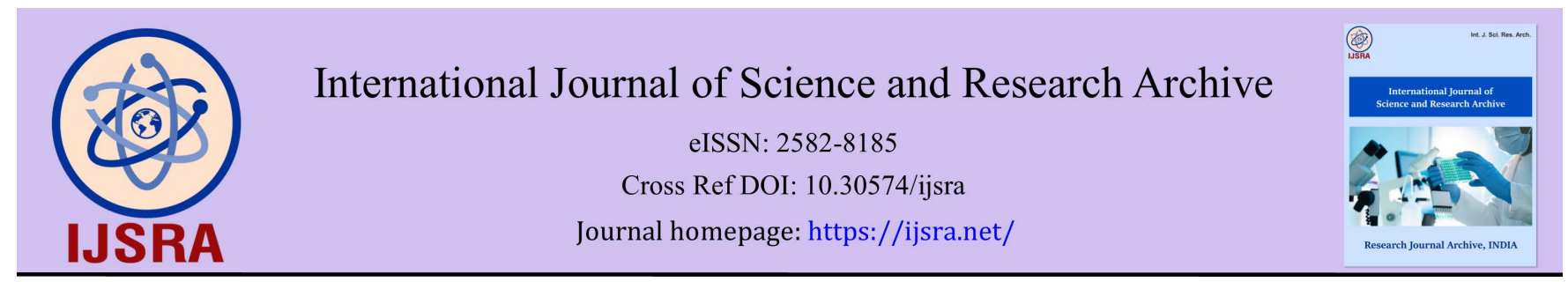

(REVIEW ARTICLE)

\title{
Childhood obesity, effects on the cardiovascular system and quality of life: The role of physical therapy
}

\author{
Konstantinos Chandolias $1{ }^{*}$ and Chrysoula Moscholouri ${ }^{2}$ \\ ${ }^{1}$ Department of Physical Therapy International Hellenic University, Greece. \\ 2 Department of Physical Therapy, University of West Attica, Greece.
}

International Journal of Science and Research Archive, 2021, 02(01), 111-115

Publication history: Received on 14 January 2021; revised on 15 February 2021; accepted on 17 February 2021

Article DOI: https://doi.org/10.30574/ijsra.2021.2.1.0032

\begin{abstract}
Childhood obesity is a global problem, with its prevalence in the developed world increasing fourfold in recent decades. Obesity in children is defined by the Body Mass Index and is responsible for many physical and psychological problems, as well as for reduced perceived quality of life. In recent years, it has become increasingly worrying that diseases affecting adult populations, such as diseases of the cardiorespiratory system, also affect children, as well as the health of adults who have been obese children. The involvement of physiotherapists in the treatment of obesity in children and adolescents can lead to a more understandable assessment and improved care and treatment of obesity.
\end{abstract}

Keywords: Childhood obesity; Cardiorespiratory system; Quality of life; Physiotherapy

\section{Introduction}

The prevalence of obesity in children and adolescents has increased 3 to 4 times in the last 3 decades in the United States. In Europe, the current prevalence of overweight or obesity in preschool children is between $8 \%$ and $13 \%$. These populations are at greater risk for endothelial dysfunction, hypertension, insulin resistance, cholecystolithiasis, hepatic non-alcoholic fatty liver disease, and respiratory and orthopedic disorders, as well as psychosocial, psychosocial, or psychiatric).

Non congenital cardiovascular disease (CVD) should be rare in children, but it is becoming more common in combination with the increase in childhood obesity. Obesity in children is defined as a body mass index (BMI) at or above the $95^{\text {th }}$ percentile for age and gender. Most troubling, however, are reports that childhood obesity is often accompanied by concomitant cardiovascular abnormalities, suggesting that the problem is not just a future or long-term risk of CVD but rather a comorbidity that requires immediate attention to prevent progressive harm. cardiorespiratory system in childhood (Cote et al, 2013). The World Health Organization defines the Healh Related Quality of life (HRQOL) as the quality of life of a person related to his physical, mental, and social well-being.

The concept of HRQOL is outside the International Classification of Disability and the Functioning of the World Health Organization. However, HRQOL measures usually include various elements of the International Classification Framework, in particular those related to activity (ie specific task performance) and participation (ie in real situations), although less frequently they include elements related to normal bodily functions (for example, neuromusculoskeletal function and pain) or contextual factors (environmental and personal). A chronic health condition can have a variety of effects on a person's functioning. A health condition can cause significant damage to bodily functions, although the individual may still be able to participate fully in community life and report HRQOL. In contrast, other individuals with

\footnotetext{
${ }^{*}$ Corresponding author: Konstantinos Chandolias

Department of Physical Therapy International Hellenic University, Greece. 
mild physical disabilities may report significant participation limitations and impaired HRQOL. Therefore, it is important to consider the impact of a health condition such as obesity on all aspects of functioning (Tsiros et al, 2009)

Therefore, children and adolescents who are obese and intend to dicrease their level of physical activity should be evaluated and monitored by physiotherapists, due to their knowledge of pathology, pharmacotherapy and exercise physiology. The systematic involvement of physiotherapists in the treatment of obesity in children and adolescents can lead to a more understandable assessment and improved care and treatment of obesity at the community level, especially in children and adolescents with increased health care needs (Hansen et al, 2015) .

The purpose of this study is the literature review on the role of the physiotherapist in the effort to improve the lives of obese children, which affects the cardiorespiratory system, and the critical evaluation of the proposed methods.

\section{Methodology}

\subsection{Selection of studies}

Included studies met the following criteria: focused on children or adolescents (up to 21 years old), estimated weight (eg weight, BMI, body fat and skin aspects) HRQOL evaluation (either parental proxy or self-report) and HRQOL report on weight status. Studies were also were included that correlated the above data with both effects on the cardiorespiratory system and the necessary physiotherapy interventions and their effect on the quality of life of children. The search was made between English and Greek spelling, dissertations, or abstracts. The keywords used were [Obese children OR adolescents] AND [Cardiorespiratory system] AND [Obese children OR adolescent] AND [Cardiorespiratory system] AND [Physiotherapy]. The databases were Pub med and Google Scholar.

\subsection{Measurement and Patterns of Obesity in Children}

Although the etiology of obesity is multifactorial, it can simply be defined as excess body fat (or fat). Adipose tissue (AT) is one of the largest organs in the body and provides protection and support to other organs and acts as endocrine tissue. Adipose tissue development is a dynamic process. The first fat cells, also called fat cells, appear during the endometrial period and continue to grow and expand throughout life. Although the increase in AT at each stage of development follows a general pattern that is specific at this stage, studies have shown that fat deposition in prenatal and infant life follows childhood and then adulthood. Many ingredients have been shown to contribute to the healthy (or unhealthy) development of AT, including genetic factors as well as prenatal and postnatal exposure to diet, lifestyle, and environmental factors. are dynamic processes, beginning in the second trimester of pregnancy and extending throughout life within a lobe through differentiated progenitor or mesenchymal cells (Orsso et al, 2020).

Anthropometry is used as a substitute measure to reflect the amount of fat. In clinical practice, BMI is expressed as a population-based percentage for age and sex, and defines either overweight (85th percentile) or obese (95th percentile) individuals, with some researchers defining severe obesity at 99th percentile. . The reference standard and limits used may affect prevalence estimates. For example, from the 2004 Canada data, the prevalence of obesity and obesity was $35 \%$ using growth curves from the World Health Organization, 26\% using the International Obesity Group, and 28\% using the Centers for Disease Control charts. US use of BMI is believed to have high specificity but low sensitivity in detecting high fat (McCrindle, 2015).

Estimating waist circumference and normalization as either age and racial percentage, waist-to-height ratio, or waistto-hip ratio have similar correlations with fat level with BMI when used alone. They may have a stronger association with the associated cardiometabolic risk when used in conjunction with BMI. It must be acknowledged that the relationship between fat and cardiovascular risk and cardiovascular outcomes is continuous and non-linear, and therefore the relative limits loosely reflect the turning points where the risk intensifies and accelerates. In addition, especially in children, cross-sectoral assessments are less important than longitudinal trends, reflex duration, and exposure intensification. Other measures that are less commonly used in clinical practice may include thickening of the skin folds, bioelectrical impedance, X-ray dual energy X-ray scan, and MRI. The location of fat deposition, especially ectopic fat, can have a significant effect on cardiometabolic risk and vascular health (Lim \& Meigs, 2014).

\section{Childhood obesity and cardiorespiratory dysfunction}

Although high fat may lead to cardiovascular disease, much of the risk is due to relevant cardiovascular risk factors. In addition to the quantitative and qualitative aspects of adipose tissue, anatomical and functional abnormalities contribute to a pathophysiological process called lipopathy. The presence of cardiometabolic disorders varies between 
individuals with the same degree of fatness, and environmental, behavioral, and genetic factors may play a role. Lipopathy can be indicated by the presence of inflammatory markers, markers of oxidative stress, altered levels of lipokines and endocrine abnormalities, especially insulin resistance, ie all conditions that have been observed in obese youth. These disorders can lead to combined dyslipidemia (high triglycerides, low-density lipoprotein [HDL], high nonHDL cholesterol, and elevated low-density lipoprotein [LDL]), which is manifested in hypertension and type 2 diabetes. obese young people. These risk factors and the oxidative stress-related inflammation associated with lipopathy contribute to endothelial dysfunction and the onset and acceleration of atherosclerosis (McCrindle, 2015).

Endothelial dysfunction is a key early stage in the development of cardiovascular disease and can be evaluated noninvasively using techniques such as flow-mediated vasodilation (FMD). Increased arterial stiffness, which is associated with an increased risk of heart disease in adults, occurs with aging, but may also be due to higher levels of certain risk factors. An indication of arterial stiffness can be obtained by measuring the compliance or distance of an arterial segment, pulse wave velocity (pulse wave velocity, PWV) or the timing and magnitude of wave reflection (pulse wave analysis) (Ayer et al , 2015).

Although it may take decades for an obese child to develop clinically evident cardiovascular disease and events, obese young people have been shown to show an acceleration of atherosclerosis. Non-invasive markers of early subclinical atherosclerosis have proven to be useful tools for determining the mechanistic correlations and the effect of interventions on children and adolescents, and there are recommendations for standardized assessment in the research environment. Cross-sectional case-control studies have confirmed that obesity and associated cardiometabolic risk factors in childhood are associated with the presence of endothelial dysfunction and are assessed using flow dilation, increased arterial stiffness, and increased incidence of stenosis (increased incidence).

\section{Physical therapy and quality of life in obese child}

Studies have shown that obese young people have poorer HRQOL than normal weight people, with studies in some obese teens suggesting they have HRQOL similar to people with cancer. Weight gain has a moderate to strong negative effect on HRQOL in pediatric populations, where reductions in HRQOL are evident as soon as the BMI is above healthy normal limits. When examining HRQOL subgroups, studies consistently report that obese young people have reduced physical activity compared to their normal weight counterparts. Aggregate analyzes and individual study findings suggest that a moderate to strong inverse relationship between weight status and physical function, with increasing weight status having a detrimental effect on physical function (Hansen et al, 2015).

The main goal in the treatment of obesity is to ensure weight loss to reduce health risks, as mentioned, to maintain a proper body weight and to avoid weight renewal. The treatment of obesity aims to reduce body weight by about 5 to $15 \%$ of the initial weight for a period of 6 months. A negative energy balance is achieved by reducing energy intake from food (limiting calorie intake with food) and / or increasing energy expenditure through muscle work (physical activity). Training programs for obese patients should include consultation with a physician to rule out contraindications to exercise. People with cardiovascular disease are advised to evaluate exercise tolerance based on the exercise test. Assistance for obese patients requires the cooperation of an interdisciplinary team, including physicians, dietitians, psychologists and physiotherapists, who should prepare an adequate training program on existing diseases (CieślińskaŚwider, 2015).

The purpose of including physical activity in the weight loss program is to increase energy expenditure and reduce excess fat, which is best achieved with continuous aerobic exercise using large muscle groups. It is important to encourage children to do regular exercise because, the habits that are cultivated during childhood are transferred to adulthood and thus to the prevention of many diseases such as cardiorespiratory disease, high blood lipids and hypertension (Khan et al, 2016).

Obese children and adolescents are less involved in an active lifestyle. For children and young people, physical activity includes individual play, group games, sports, physical work, leisure, physical education or scheduled exercise, as part of family, school and community activities. The WHO recommends that children aged 5 to 17 should be physically active for at least one hour at a moderate intensity each day. Most daily physical activity should be aerobic. Intense activities should be integrated, including those that strengthen muscles and bones at least 3 times a week (WHO, 2010).

Exercise amounts longer than 60 minutes provide additional health benefits. Sedentary behavior in children, 5 to 18 years old, seems to be associated with weight gain, decreased aerobic capacity, lower self-esteem and learning ability. It is also recommended that children under 5 years of age be physically active for at least 180 minutes a day throughout the day. For children under 5 years of age the effect of sedentary behavior seems to be associated with increased weight, 
and negative cognitive and psychosocial development, events that also contribute to the reduction of perceived quality of life (Pate et al, 2008).

Physical exercise in children 5-17 years old contributes to the formation of a healthy cardiorespiratory system, to positive results in blood lipids, to the reduction of body weight and to a healthy musculoskeletal system. Physical activity also has a positive effect on mental health, improving self-control in obese children, excessive symptoms of depression and anxiety, and promoting improved self-esteem (Rauner et al, 2013).

Overweight / obese children have the same health benefits from physical activity as normal weight children. However, musculoskeletal problems and symptoms or other obstacles can limit the time of physical activity. Low physical activity seems to be a risk factor for developing overweight / obesity and physical activity therefore prevents the development of obesity. Aerobic exercise reduces the amount of body fat. Even though the scientific data on the frequency, duration and intensity of physical activity is limited, a healthy physically active lifestyle combined with a balanced diet can reduce the risk of childhood obesity and contribute to healthy growth. . There is evidence that physiotherapy sessions and multidisciplinary interventions, including physiotherapy, are significantly effective in improving activity levels and body mass index (Nooijen et al, 2017).

As experts in movement and exercise, and with full knowledge of functional anatomy and pathology and its effects on all systems, physiotherapists have the specific ability and know-how to promote, guide, adapt and manage physical activity, especially in children and young people with chronic health conditions. Physiotherapy is traditionally delivered in a variety of settings: communities, including care centers and individual homes and space in primary care and hospital. However, today we face new challenges related to epidemic levels of physical inactivity among children and young people. It is therefore necessary to identify other areas in which physiotherapy should be provided (World Confederation for Physical Therapy, 2018).

Physical education programs in schools have been recognized as an important tool for raising awareness of physical activity that improves health. There is evidence that the quality of such programs is important for the outcome of physical education training programs. With their extensive knowledge of promoting, guiding, and managing physical activity for children with disabilities, physiotherapists are excellent partners in developing, implementing, and monitoring high quality physical education programs in schools. In addition, physiotherapists have a specific ability to demonstrate the relationship between physical activity and health, and work with defined physical activity to achieve health and well-being (Heckman et al, 2010).

\section{Conclusion}

Overweight and obesity in childhood and adolescence is a major and growing public health problem worldwide. High fat in youth is indicated in clinical practice with the body mass index at appropriate percentages set for age and gender, although waist measurement may be a further tool. High fat can lead to obesity in young people, with associated increases in inflammation and oxidative stress, changes in lipokines and endocrinopathy. This manifests as cardiometabolic risk factors in patterns similar to those seen in obese adults. Obesity and cardiometabolic risk factors have been shown to be associated with vascular changes indicative of early atherosclerosis and ventricular hypertrophy, dilatation, and dysfunction. These cardiovascular effects are evident in youth, but childhood obesity is also predictive of similar effects in adulthood.

Obesity is a complex disorder with many related complications, and even if the obese child achieves a healthy adult weight, he or she is still at risk of significant morbidity. Overweight children are more likely to grow up as overweight adults. Obesity during adolescence has been found to increase stereotypes and can lead to low self-esteem and depression. Psychological problems, combined with reduced ability to exercise, lead to lower health-related quality of life.

All of the above lead to the conclusion that the adoption of a healthy lifestyle and weight loss is a necessary condition for increasing the quality of life in obese children.

Fitness training is required to avoid complications that may occur due to obesity. Training and strengthening exercises are required to increase endurance in obese children and reduce body fat. A physically active lifestyle as a child promotes a healthy lifestyle and disease prevention. Obese children are less physically fit than normal weight children and physical inactivity is a major factor in childhood obesity. Regular physical activity has been linked to maintaining optimal metabolic function and plays an important role in a child's social and mental development. 
Physiotherapists, who have the ability to promote, guide, and manage physical activity for children with disabilities, can be excellent partners in developing, implementing, and monitoring high-quality physical education programs in schools, homes, and community structures. In addition, physiotherapists have a specific ability to demonstrate the relationship between physical activity and health, and work with defined physical activity to achieve health and well-being.

\section{Compliance with ethical standards}

\section{Acknowledgments}

The authors would like to acknowledge International Hellenic University and the MSc program Pediatric Physical Therapy for the opportunity to conduct this review.

\section{Disclosure of conflict of interest}

Both authors report no conflict of interest

\section{References}

[1] Ayer J, Charakida M, Deanfield JE, Celermajer DS. Lifetime risk: childhood obesity and cardiovascular risk. European Heart Journal. 2015; 36(22): 1371-1376.

[2] Cieślińska-Świder J. Physiotherapy in the comprehensive treatment of obesity. Physiotherapy and Health Activity. 2015; 23.

[3] Cote AT, Harris KC, Panagiotopoulos C, Sandor GGS, Devlin AM. 2013. Childhood Obesity and Cardiovascular Dysfunction. Journal of the American College of Cardiology. 2013; 62(15): 1309-1319.

[4] Hansen D, Hens W, Peeters S, Wittebroot C, Van Ussel S, Verleyen D, Vissers D. Physical Therapy as Treatment for Childhood Obesity in Primary Health Care: Clinical Recommendation From AXXON (Belgian Physical Therapy Association). Physical Therapy. 2015; 96(6).

[5] Heckman JJ, Moon SH, Pinto R, Savelyev PA, Yavitz A. The Rate of Return to the High/Scope Perry Preschool Program. Journal of public economics. 2010; 94(1-2): 114-128.

[6] Khan S, Malawade M, Kularni S, Khatri S. Effectiveness of Fitness Training in Obese Children. International Journal of Physiotherapy and Research. 2016; 4: 1498-1502.

[7] Lim S, Meigs JB. Links between ectopic fat and vascular disease in humans. Arteriosclerosis, thrombosis, and vascular biology. 2014; 34(9): 1820-1826.

[8] Mc Crindle BW. Cardiovascular Consequences of Childhood Obesity. Canadian Journal of Cardiology. 2015; 31(2): 124-130.

[9] Nooijen CF, Galanti MR, Engström K, Möller J, Forsell Y. Effectiveness of interventions on physical activity in overweight or obese children: a systematic review and meta-analysis including studies with objectively measured outcomes. Obesity Reviews. 2017; 18(2): 195-213.

[10] Orsso CE, Colin-Ramirez E, Field CJ, Madsen KL, Prado CM, Haqq AM. Adipose Tissue Development and Expansion from the Womb to Adolescence: An Overview. Nutrients. 2020; 12 (2735).

[11] Pate RR, O'Neill JR, Lobelo F. The evolving definition of "sedentary". Exercise and Sport Sciences Reviews. 2008; 36(4): 173-178.

[12] Rauner A, Mess F, Woll A. The relationship between physical activity, physical fitness and overweight in adolescents: a systematic review of studies published in or after 2000. BMC pediatrics. 2012; 13.

[13] Tsiros M, Olds T, Buckley JD, Grimshaw P, Brennan L, Walkley J, Hills AP, Howe PRC, Coates AM. Health-related quality of life in obese children and adolescents. International Journal of Obesity. 2009; 33(4): 387-400.

[14] WHO. Global recommendations on physical activity for health, Genève, Switzerland: World Health Organization. 2010.

[15] World Confederation for Physical Therapy. Promoting physical activity in children, the role of Physiotherapists, European Region: EU Platform for Action on Diet, Physical Activity and Health. 2018. 